\title{
Giant modification of atomic transitions probabilities induced by magnetic field: forbidden transitions become predominant
}

\author{
A. Sargsyan ${ }^{1}$, A. $\operatorname{Tonoyan}^{1}$, G. Hakhumyan ${ }^{1}$, A. $\operatorname{Papoyan}^{1}$, E. Mariotti ${ }^{2}$, D. Sarkisyan ${ }^{1}$ \\ ${ }^{1}$ Institute for Physical Research, NAS of Armenia, Ashtarak-2, 0203, Armenia \\ ${ }^{2}$ CNISM - Department of Physical, Earth and Environmental Sciences, University of Siena, Via Roma \\ 56, 53100 Siena, Italy
}

\begin{abstract}
Magnetic field-induced giant modification of probabilities for seven components of $6 \mathrm{~S}_{1 / 2}\left(\mathrm{~F}_{\mathrm{g}}=3\right)$ $\rightarrow 6 \mathrm{P}_{3 / 2}\left(\mathrm{~F}_{\mathrm{e}}=5\right)$ transition of $\mathrm{Cs} \mathrm{D}_{2}$ line forbidden by selection rules is observed experimentally for the first time. For the case of excitation with circularly-polarized laser radiation, the probability of $\mathrm{F}_{\mathrm{g}}=3, \mathrm{~m}_{\mathrm{F}}=-3 \rightarrow \mathrm{F}_{\mathrm{e}}=5, \mathrm{~m}_{\mathrm{F}}=-2$ transition becomes the largest among 25 transitions of $\mathrm{F}_{\mathrm{g}}=3 \rightarrow \mathrm{F}_{\mathrm{e}}=2,3,4,5$ group in a wide range of magnetic field $200-3200 \mathrm{G}$. Moreover, the modification is the largest among $\mathrm{D}_{2}$ lines of alkali metals. A half-wave-thick cell (length along the beam propagation axis $\mathrm{L}=426 \mathrm{~nm}$ ) filled with $\mathrm{Cs}$ has been used in order to achieve subDoppler resolution which allows for separating the large number of atomic transitions that appear in the absorption spectrum when an external magnetic field is applied. For B $>3 \mathrm{kG}$ the group of seven transitions $\mathrm{Fg}=3 \rightarrow \mathrm{Fe}=5$ is completely resolved and is located at the high frequency wing of $\mathrm{Fg}=3 \rightarrow \mathrm{Fe}=2,3,4$ transitions. The applied theoretical model very well describes the experimental curves.
\end{abstract}

\section{INTRODUCTION}

Alkali atoms are widely used in atomic physics due to simplicity of electronic structure and strong atomic transitions from the ground state with wavelengths in visible and nearinfrared, where diode lasers with good parameters are available. Cesium atoms are widely used in laser cooling experiments, information storage, spectroscopy, magnetometry, laser frequency stabilization etc. [1-3]. That is why any knowledge of the behavior of Cs atomic transitions, particularly, in an external magnetic field is of high importance. It is well known that in quite moderate magnetic field $B$ the splitting of atomic energy levels to Zeeman sublevels deviates from the linear behavior, and the atomic transition probabilities undergo significant changes [46]. The most simple and straightforward technique to study such modification of atomic transitions (whose frequency distance belongs to optical domain) is laser spectroscopy of atoms contained in an atomic vapor cell. For $B$ up to $\cong 1000 \mathrm{G}$, the split Zeeman transitions remain overlapped because of Doppler broadening, and sub-Doppler techniques have to be implemented in order to spectrally resolve and study transition probability of individual transition components [7].

As it was demonstrated earlier, strong narrowing in absorption spectrum can be attained with the use of an atomic vapor cell of half-wavelength thickness $(L=\lambda / 2$, where $\lambda$ is the resonant wavelength of laser radiation, $L=426 \mathrm{~nm}$ for the case of $C s \mathrm{D}_{2}$ line) [8-11]. Particularly, the absorption linewidth for $\mathrm{Cs}_{2}$ line reduces to $\cong 100 \mathrm{MHz}$ (FWHM), as opposed to $\cong 400 \mathrm{MHz}$ in an ordinary cell. Moreover, the absorption lines for $L=\lambda / 2$ exhibit Voigt profile (a convolution of Lorentzian and Gaussian profiles) with sharp (nearly Gaussian) peak, which allows separation of closely spaced individual transitions and study their transition probabilities in an external magnetic field. In addition, the $\lambda / 2$-method is tolerant against $10 \%$ deviation of thickness (weak influence on the absorption linewidth). These benefits make it convenient to use $\lambda / 2$-method for studies of closely spaced individual atomic transition components in a magnetic field. 
In this letter we present, for the first time, the results of experimental and theoretical studies showing a giant transition probability modification for $\mathrm{Cs}_{2} \mathrm{D}_{2}$ line $6 \mathrm{~S}_{1 / 2}, \mathrm{~F}_{\mathrm{g}}=3 \rightarrow 6 \mathrm{P}_{3 / 2}$, $\mathrm{F}_{\mathrm{e}}=5$ transition induced by a magnetic field varied in a wide range up to $3.5 \mathrm{kG}$. It should be remembered that, according to the electric dipole selection rules in zero B field, only transitions with a change of atomic total angular momentum $\Delta \mathrm{F}=\mathrm{F}_{\mathrm{g}}-\mathrm{F}_{\mathrm{e}}=0, \pm 1$ are allowed, while $\mathrm{F}_{\mathrm{g}}=3 \rightarrow \mathrm{F}_{\mathrm{e}}=5$ transitions are forbidden. To the best of our knowledge, there are only few articles where such type of transitions have been quantitatively studied [4,6,11].

\section{EXPERIMENTAL}

Nanometric Thin Cells (NTCs) filled with Cs have been used in our experiment, because of their peculiar properties which allow a) to directly obtain sub-Doppler spectra without application of nonlinear techniques and therefore b) to resolve even complicated spectra, as already demonstrated in many papers published in the last few years after their introduction in laser spectroscopy [8].

The general design of NTC is similar to that described in ref.12. Compact oven has been used to set the needed temperature regime. The temperature was set to $100{ }^{\circ} \mathrm{C}$, which corresponds to number density of isotopically pure ${ }^{133} \mathrm{Cs}$ atoms $N \cong 10^{13} \mathrm{~cm}^{-3}$. Adjustment of needed vapor column thickness without variation of thermal conditions was attained by smooth vertical translation of the cell+oven assembly.

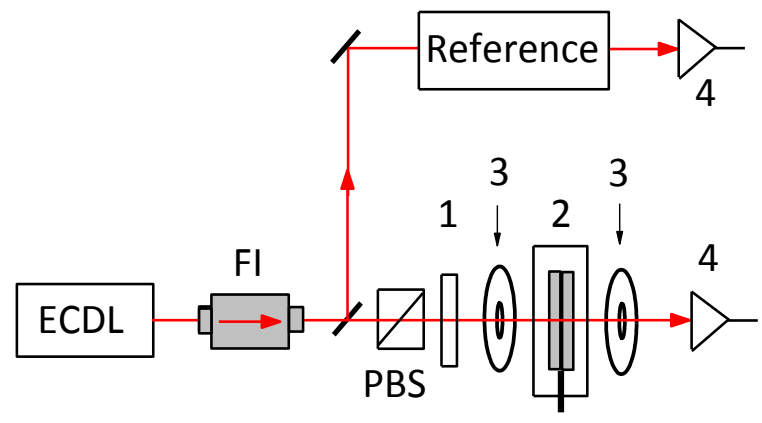

Fig.1. Sketch of the experimental setup. ECDL- External Cavity Diode Laser; FIFaraday Isolator; $1-\lambda / 4$ plate; $2-$ NTC in the oven; PBS- Polarizing Beam Splitter; 3permanent ring magnets; 4- photodetectors; Reference- auxiliary Cs NTC providing $B=0$ reference spectrum.

Schematic diagram of the optical part of experimental setup is shown in Fig.1. A circularly polarized laser radiation beam $\left(\lambda=852 \mathrm{~nm}, P_{L}=5 \mathrm{~mW}, \Delta v_{L}=1 \mathrm{MHz}\right)$ resonant with Cs $\mathrm{D}_{2}$ line was focused $(\varnothing=0.5 \mathrm{~mm}$ ) onto a Cs NTC with a vapor column of thickness $L=\lambda / 2$ at normal incidence angle. The $8 \mathrm{~mm}$-thick assembly of the oven with main NTC inside was placed between two permanent ring magnets (whose axis is directed along the laser radiation propagation direction $\mathbf{k}$ ) with gradually adjustable spacing providing controllable longitudinal $B$ field (for details, see [6]). Extremely small thickness of NTC is once more advantageous for application of very strong magnetic field with the use of permanent magnets otherwise unusable because of strong inhomogeneity: in NTC, the variation of the $B$-field inside the cell is several orders less than the applied $B$ value [13]. To record transmission and fluorescence spectra, the laser radiation was linearly scanned within up to $15 \mathrm{GHz}$ spectral region covering the studied group of transitions. The nonlinearity of the scanned frequency $(<1 \%$ throughout the spectral range) was monitored by simultaneously recorded transmission spectra of a Fabry-Pérot etalon (not shown). About $30 \%$ of the pump power was branched to an auxiliary Cs NTC with thickness $L=\lambda / 2$ providing reference absorption spectrum for $B=0$. All the spectra were detected by photodiodes with amplifiers followed by a four channel digital storage oscilloscope Tektronix TDS 2014B. 


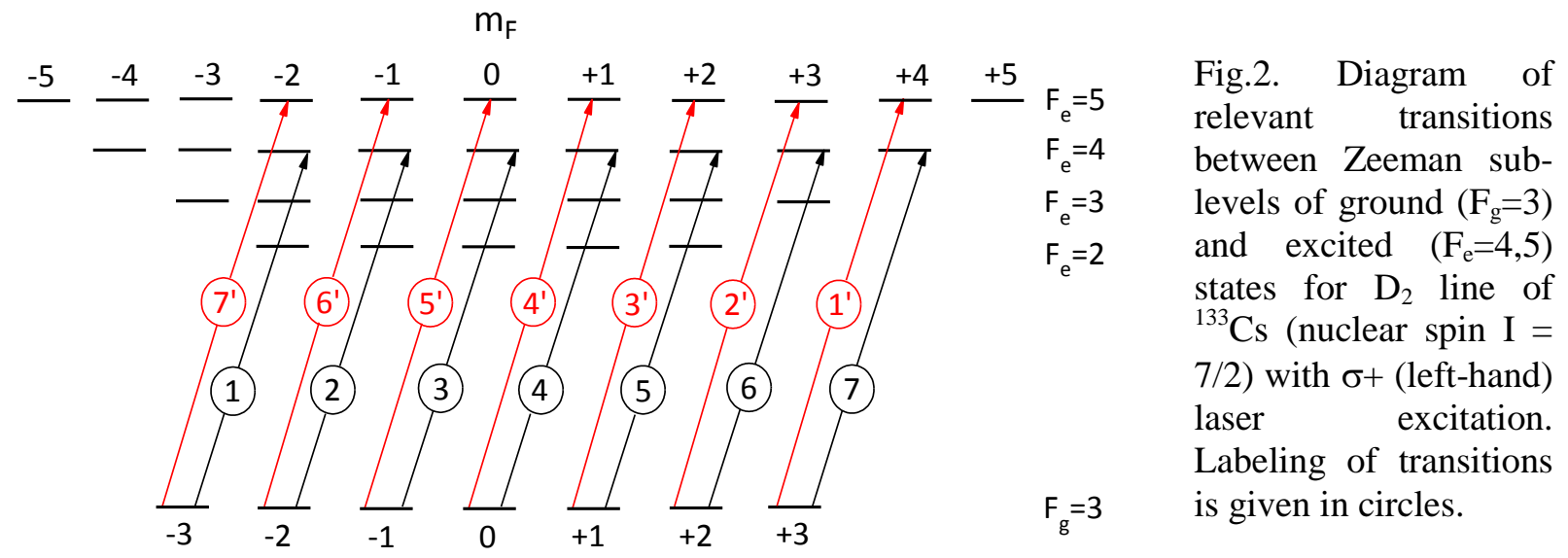

The diagram of relevant $\sigma^{+}$components $\left(\Delta \mathrm{m}_{\mathrm{F}}=+1\right)$ of $\mathrm{Cs} \mathrm{D}_{2}$ line transitions $\mathrm{F}_{\mathrm{g}}=3 \rightarrow$ $\mathrm{F}_{\mathrm{e}}=4$ labeled $1-7$, and $\mathrm{F}_{\mathrm{g}}=3 \rightarrow \mathrm{F}_{\mathrm{e}}=5$ labeled 1' - 7' is shown in Fig.2. The transitions 1' - 7' are forbidden for zero magnetic field because of selection rule $\Delta \mathrm{F}=0, \pm 1$. The transitions $\mathrm{F}_{\mathrm{g}}=3 \rightarrow$ $\mathrm{F}_{\mathrm{e}}=2,3$ are not shown, since for $B>500 \mathrm{G}$ their probabilities strongly reduce, and these transitions are practically not detectable in the absorption spectrum.

The recorded absorption spectrum of Cs NTC with thickness $L=\lambda / 2$ for $\sigma^{+}$laser excitation $\left(P_{L}=10 \mu \mathrm{W}\right)$ and $B=920 \mathrm{G}$ is shown in Fig.3. The fourteen above labeled transition components appear with $\cong 100 \mathrm{MHz}$ linewidth, thus being completely frequency resolved except for transitions 5, 6 and 7' resolved partially, and 6' and 7, which are fully overlapped. As it is seen from the inset the amplitudes of $\mathrm{Fg}=3, \mathrm{~m}_{\mathrm{F}}=-3 \rightarrow \mathrm{Fe}=5, \mathrm{~m}_{\mathrm{F}}=-2$ and $\mathrm{Fg}=3, \mathrm{~m}_{\mathrm{F}}=-2 \rightarrow \mathrm{Fe}=5$, $\mathrm{m}_{\mathrm{F}}=-1$ transitions (labeled $7^{\prime}$ and $6^{\prime}$, respectively) are the largest ones among the group of fourteen lines.

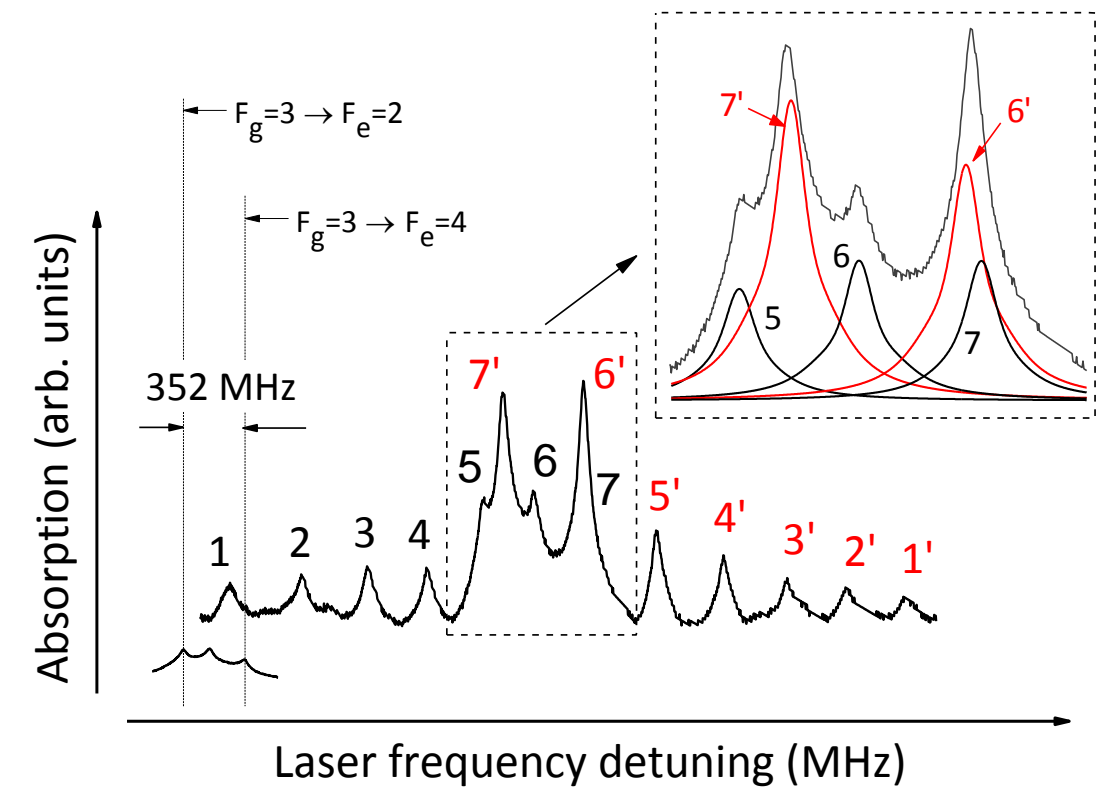

Fig.3. Absorption spectrum of Cs NTC with $L=\lambda / 2$ for $B=$ $920 \mathrm{G}$ and $\sigma^{+}$laser excitation. For labeling of transitions, see Fig.2. The bottom-left curve is absorption spectrum of the reference NTC showing positions of $\mathrm{F}_{\mathrm{g}}=3 \rightarrow \mathrm{F}_{\mathrm{e}}=2,3,4$ transitions for $B=0$. The inset shows an expanded view of the part of the experimental results limited by the dashed rectangle and the corresponding fit of the individual lines.

The fitting is justified thanks to the following advantageous property of the $\lambda / 2$-method: for the case of a weak absorption, the absorption coefficient of an individual transition component $A$ is proportional to $\sigma N L$, where $\sigma$ is the absorption cross-section proportional to $d^{2}$ ( $d$ being the matrix element of the dipole moment), $N$ is atomic density, and $L$ is the thickness. Measuring the ratio of $A_{i}$ values for different individual transitions, it is straightforward to estimate their relative probabilities (line intensities). As is seen from the inset, the amplitudes of $7^{\prime}$ and $6^{\prime}$ transitions forbidden at $B=0$ are the largest among all fourteen transitions. 


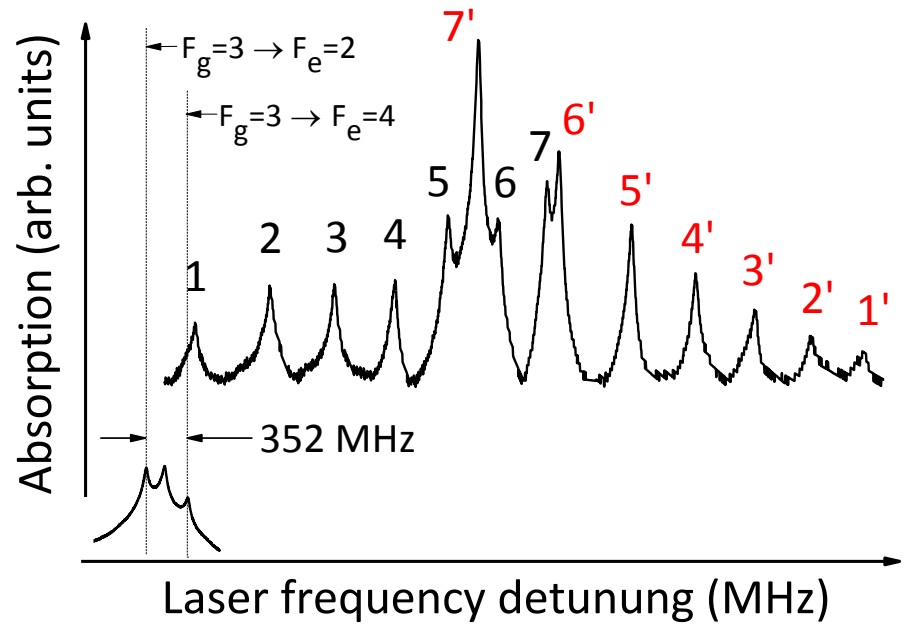

Fig.4. Absorption spectrum of Cs NTC with $L=\lambda / 2$ for $B=$ $1450 \mathrm{G}$ and $\sigma^{+}$laser excitation. For labeling of transitions, see Fig.2. The bottom-left curve is absorption spectrum of the reference NTC showing positions of $\mathrm{F}_{\mathrm{g}}=3 \rightarrow \mathrm{F}_{\mathrm{e}}=2,3,4$ transitions for $B=0$.

Further increase of magnetic field to $B=1450 \mathrm{G}$ results in better resolution of individual transitions (Fig.4). Complete separation of all the fourteen transition components occurs at $B>$ $3000 \mathrm{G}$. The absorption spectrum for $B=3450 \mathrm{G}$ and otherwise invariable experimental conditions as in Fig.3 and Fig. 4 is presented in Fig.5. Here also the groups of seven transitions 1 - $7\left(\mathrm{~F}_{\mathrm{g}}=3 \rightarrow \mathrm{F}_{\mathrm{e}}=4\right)$ and $1^{\prime}-7^{\prime}\left(\mathrm{F}_{\mathrm{g}}=3 \rightarrow \mathrm{F}_{\mathrm{e}}=5\right)$ are completely separated as marked by dashed rectangles. In addition, two transition components from the group $F_{g}=4 \rightarrow F_{e}=5$ appear to be located on the low frequency side of the spectrum.

Noteworthy that the intensity of the transition component labeled $7^{\prime}$ is the largest among all the 25 atomic transitions of $\mathrm{F}_{\mathrm{g}}=3 \rightarrow \mathrm{F}_{\mathrm{e}}=2,3,4,5$ group for the magnetic field range $250 \mathrm{G}<B$ $<3200 \mathrm{G}$.

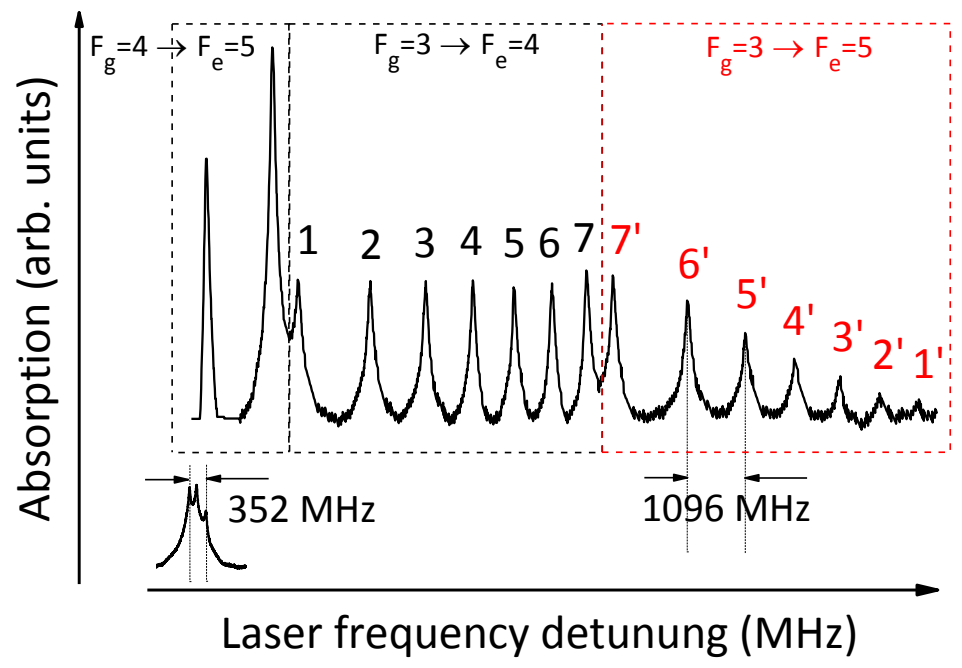

Fig.5. Absorption spectrum of Cs NTC with $L=\lambda / 2$ for $B=3450$ $\mathrm{G}$ and $\sigma^{+}$laser excitation. For labeling of transitions, see Fig.2. The bottom-left curve is absorption spectrum of the reference NTC showing positions of $\mathrm{F}_{\mathrm{g}}=3 \rightarrow \mathrm{F}_{\mathrm{e}}=2,3,4$ transitions for $B=0$. All the individual Zeeman transitions 1-7 and $1^{\prime}-7^{\prime}$ are completely resolved. Two shifted components of $\mathrm{F}_{\mathrm{g}}=4 \rightarrow \mathrm{F}_{\mathrm{e}}=5$ transition appear at the low frequency region.

\section{THEORETICAL MODEL AND DISCUSSION}

Simulations of magnetic sublevel energy and relative transition probabilities for $\mathrm{F}_{\mathrm{g}}=3 \rightarrow$ $\mathrm{F}_{\mathrm{e}}=2,3,4,5$ transitions of $\mathrm{Cs} \mathrm{D}_{2}$ line are well known, and are based on the calculation of dependence of the eigenvalue and eigenvector of the Hamilton matrix on magnetic field for the full hyperfine structure manifold $[2,4,5]$. The dependence of frequency shifts of transitions $1^{\prime}-7^{\prime}$ and 1 - 7 on magnetic field relative to position of $\mathrm{F}_{\mathrm{g}}=3 \rightarrow \mathrm{F}_{\mathrm{e}}=4$ transition at $B=0$ for the case of $\sigma^{+}$excitation is shown in Fig.6. Good agreement of theory and experiment is observed throughout the whole explored range of $B$-field (up tp $3500 \mathrm{G}$ ). 


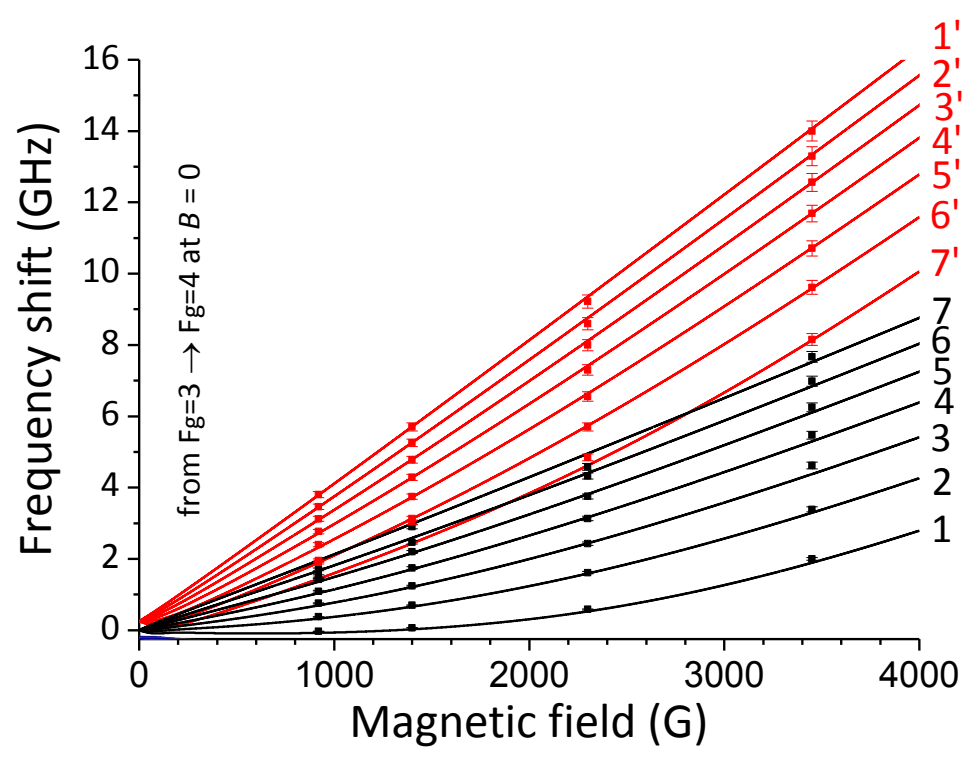

Fig.6. Magnetic field dependence of frequency shift for atomic transition labeled $1^{\prime}-7^{\prime}$ and $1-7$ relative to the position of $\mathrm{F}_{\mathrm{g}}=3$ $\rightarrow \mathrm{F}_{\mathrm{e}}=4$ transition at $B=0$. Black squares: experimental results (inaccuracy $\cong 2 \%$ ); solid curves: calculated dependence.

The calculated dependence of $1^{\prime}-7^{\prime}$ and 1 - 7 transition probabilities (absorption amplitudes) on magnetic field for the case of $\sigma^{+}$laser excitation are shown in Fig.7.

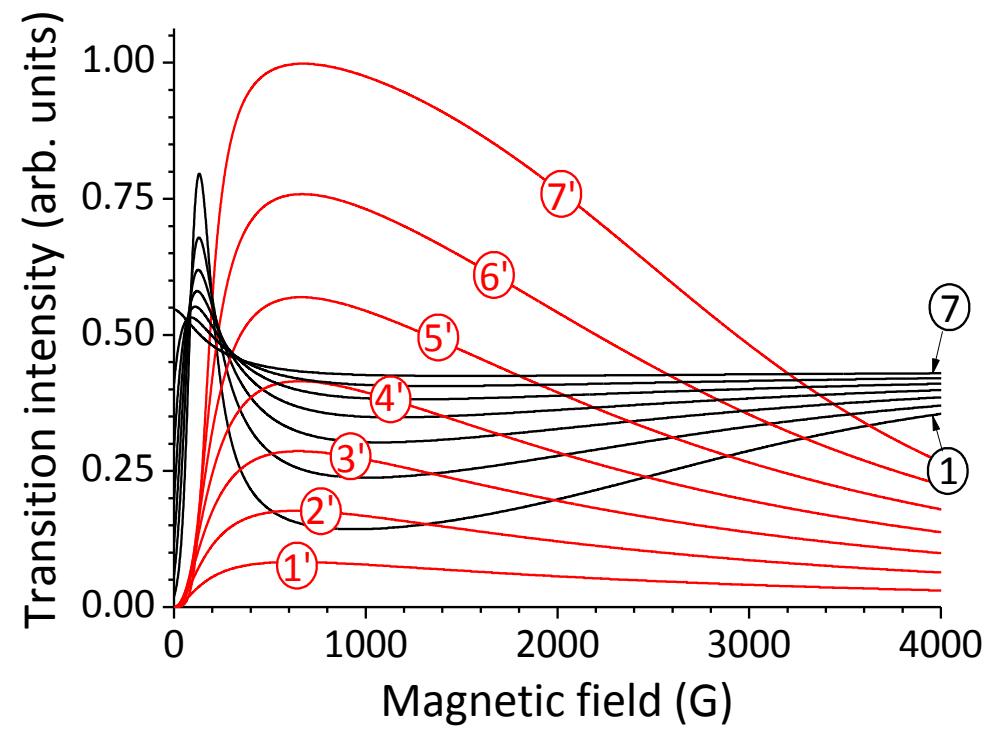

Fig.7. Calculated probabilities (absorption amplitudes) of transitions $1^{\prime}-7^{\prime}$ and $1-7$ versus magnetic field for $\sigma^{+}$laser excitation.

Since the absolute value of the absorption amplitude $A$ depends on parameters of the experiment (laser intensity, atomic density, etc.), it is expedient to present also the $B$-field dependence of the ratio of absorption amplitudes $A_{i}^{\prime}$ of $1^{\prime}-7^{\prime}$ transitions to absorption amplitude $A_{7}$ of the transition 7. The latter is the strongest in the $\mathrm{F}_{\mathrm{g}}=3 \rightarrow \mathrm{F}_{\mathrm{e}}=4$ group; moreover, the absorption amplitude $A_{7}$ is nearly constant in a wide range $250 \mathrm{G}<B<4000 \mathrm{G}$ (see Fig.7), which makes it convenient to use as a reference. The theoretical ratio $A_{i}^{\prime} / A_{7}$ versus $B$-field is plotted in Fig.8 together with experimental results (the ratio is easily measurable). The dashed line marks the unity ratio. As it is seen from Fig. $8, A_{7}^{\prime} / A_{7}>1$ holds in a wide range $200 \mathrm{G}<B$ $<3200 \mathrm{G}$, and the maximum value of the ratio is 2.3 . 


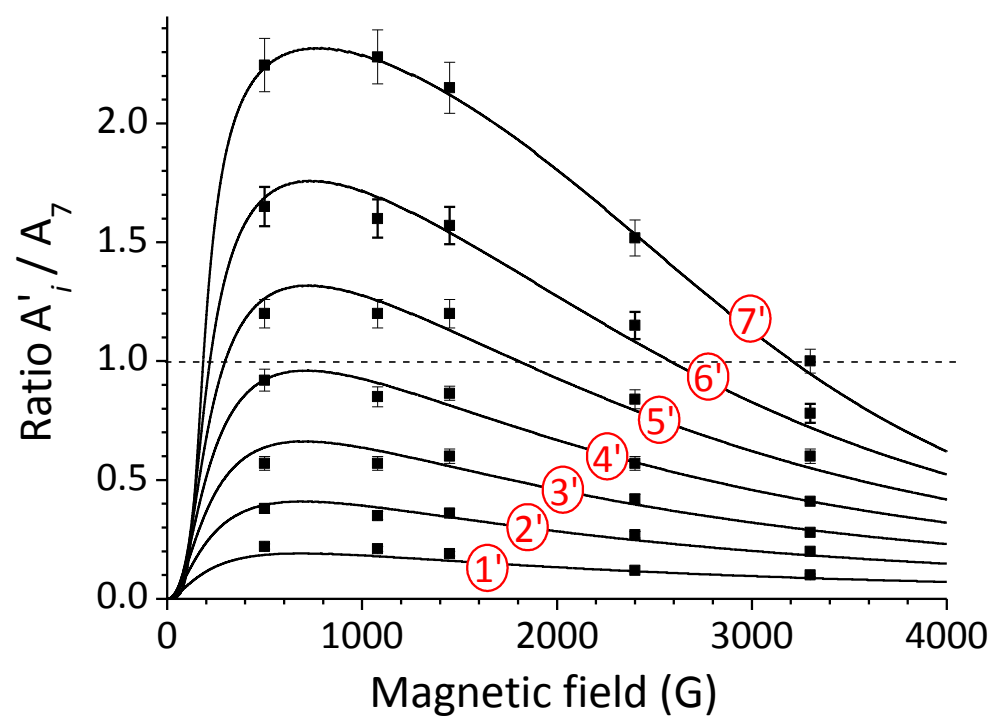

Fig.8. Ratio of absorption amplitudes $A_{i}^{\prime}$ (transitions $1^{\prime}$ - 7') to $A_{7}$ versus $B$ for the case of $\sigma^{+}$excitation. The dashed line shows the range of magnetic field where the ratio $A_{i}$ / $A 7=1$.

Thus, the transition $7^{\prime}$, as well as $6^{\prime}$ and $5^{\prime}$, which are forbidden for $B=0$ undergo giant modification under the influence of magnetic field, becoming predominant over the initially allowed transitions 1 - 7. It is worth noting that the maximum value of transition probability for $7^{\prime}$ transition reaches $76 \%$ of the probability for $\mathrm{F}_{\mathrm{g}}=4, \mathrm{~m}_{\mathrm{F}}=+4 \rightarrow \mathrm{F}_{\mathrm{e}}=5, \mathrm{~m}_{\mathrm{F}}=+5$ transition, which is the strongest among all the 54 Zeeman transitions of $\mathrm{Cs}_{2}$ line.

We want also to stress that another group of transitions forbidden at $B=0, \mathrm{~F}_{\mathrm{g}}=4 \rightarrow \mathrm{F}_{\mathrm{e}}=2$ ( 5 transition components for the case of $\sigma^{+}$excitation), also exhibits modification induced by magnetic field. However, its maximum probability value achieved at $B=60 \mathrm{G}$ is over 30 times smaller than the maximum probability value for $\mathrm{F}_{\mathrm{g}}=3 \rightarrow \mathrm{F}_{\mathrm{e}}=5$.

The decoupling of the total angular momentum $J$ and the nuclear momentum $I$ (hyperfine Paschen-Back regime) for Cs atom occurs for $B>>B_{0}=A_{H F S} / \mu_{B} \cong 1700 \mathrm{G}$, where $A_{H F S}=h \times 2.3$ $\mathrm{GHz}$ is the magnetic dipole hyperfine constant for $6 \mathrm{~S}_{1 / 2}$, and $\mu_{B}$ is the Bohr magneton. In this case the splitting of transitions is described by the projections $m_{J}$ and $m_{I}$ [13-18]. At $B>6000 \mathrm{G}$, sixteen transitions are observable in the absorption spectrum: by 8 starting from the ground states $6 \mathrm{~S}_{1 / 2}, \mathrm{~m}_{\mathrm{J}}=-1 / 2$ and and $6 \mathrm{~S}_{1 / 2}, \mathrm{~m}_{\mathrm{J}}=+1 / 2$. For $B>3000 \mathrm{G}$ and $\sigma^{+}$excitation, the group of $\mathrm{F}_{\mathrm{g}}=3 \rightarrow$ $\mathrm{F}_{\mathrm{e}}=5$ transitions it is always located at the high frequency side of $\mathrm{F}_{\mathrm{g}}=3 \rightarrow \mathrm{F}_{\mathrm{e}}=2,3,4$ transitions, with line intensities monotonically reducing and completely vanishing at $B>9000 \mathrm{G}$.

It is interesting to compare the evolution of maximum probability value of magnetic field-induced $\mathrm{Cs} \mathrm{F}_{\mathrm{g}}=3 \rightarrow \mathrm{F}_{\mathrm{e}}=5$ transition normalized to the strongest $\mathrm{D}_{2}$ transition with the corresponding values for $\mathrm{D}_{2}$ line transitions forbidden at $B=0$ for other alkali metal atoms. The theoretical calculations show that for $\mathrm{Rb}$ transitions ${ }^{85} \mathrm{Rb} 5 \mathrm{~S}_{1 / 2}, \mathrm{~F}_{\mathrm{g}}=2 \rightarrow 5 \mathrm{P}_{3 / 2}, \mathrm{~F}_{\mathrm{e}}=4$ and ${ }^{87} \mathrm{Rb}$ $5 \mathrm{~S}_{1 / 2}, \mathrm{~F}_{\mathrm{g}}=1 \rightarrow 5 \mathrm{P}_{3 / 2}, \mathrm{~F}_{\mathrm{e}}=3$, the maximum value is less 1.10 and 1.36 times, correspondingly. Also for $3 \mathrm{~S}_{1 / 2}, \mathrm{Fg}=1 \rightarrow 3 \mathrm{P}_{3 / 2}, \mathrm{~F}_{\mathrm{e}}=3$ transitions of $\mathrm{Na}$ and $4 \mathrm{~S}_{1 / 2}, \mathrm{~F}_{\mathrm{g}}=1 \rightarrow 4 \mathrm{P}_{3 / 2}, \mathrm{~F}_{\mathrm{e}}=3$ of $\mathrm{K}$ the maximum values are less (1.31 and 1.33 times, correspondingly). Thus, the modification of the probabilities for cesium $\mathrm{F}_{\mathrm{g}}=3 \rightarrow \mathrm{F}_{\mathrm{e}}=5$ transitions is the strongest among all the alkali atoms.

\section{CONCLUSION AND OUTLOOK}

Giant modification of probabilities of $\mathrm{Cs} \mathrm{D}_{2}$ line transitions $6 \mathrm{~S}_{1 / 2}, \mathrm{~F}_{\mathrm{g}}=3 \rightarrow 6 \mathrm{P}_{3 / 2}, \mathrm{~F}_{\mathrm{e}}=5$ induced by magnetic field $B$ has been studied both the experimentally and theoretically. It has been revealed for the first time that the absorption intensity for $F_{g}=3, m_{F}=-3 \rightarrow F_{e}=5, m_{F}=-2$ transition becomes the largest among all the 25 Zeeman transitions of the $F_{g}=3 \rightarrow F_{e}=2,3,4,5$ manifold for the case of $\sigma^{+}$excitation in a wide range of magnetic field $200-3200 \mathrm{G}$. It is demonstrated that utilization of a half-wavelength-thick cell filled with Cs, favorable for strong reduction of Doppler broadening of absorption lines, allows to quantitatively study the frequency 
positions and modification of individual transition probabilities. As a result of special interest, for $B>3000 \mathrm{G}$ all the seven transitions of $\mathrm{F}_{\mathrm{g}}=3 \rightarrow \mathrm{F}_{\mathrm{e}}=5$ group are completely separated. Calculated theoretical curves for transition frequency shifts and modification of probabilities induced by magnetic field for all the transitions under study show very good coincidence with the experimental results.

The largest probabilities of "forbidden" transitions labeled 7', 6' and 5' in a wide range of magnetic field (see Fig.8) make them attractive for formation of sub-natural Electromagnetically Induced Transparency resonances in new frequency regions [3,19] (for the realization of this experiment, the coupling laser frequency must be in resonance with $\mathrm{F}_{\mathrm{g}}=4 \rightarrow \mathrm{F}_{\mathrm{e}}=5$, which is easy to realize). It should be noted that $\lambda / 2-\mathrm{M}$ method can be implemented successfully to study forbidden transitions of $\mathrm{D}_{2}$ lines of other alkalis, as $\mathrm{Rb}, \mathrm{K}$ and $\mathrm{Na}$.

\section{Acknowledgements}

The authors are grateful to A. Sarkisyan for his valuable participation in the development and fabrication of the NTC. This work has received partial funding from the EU Seventh Framework Programme (FP7/2007-2013) under Grant Agreement $n^{\circ}$ 295264-COSMA. A.S., G.H. and D.S. acknowledge financial support from the State Committee Science, MES of Armenia (Research Project № 13-1C029).

\section{REFERENCES}

[1]. D. Budker, D.F. Kimball, D.P. DeMille, Atomic Physics, An Exploration through Problems and Solutions, Second Edition (Oxford University Press, ISBN 978-0-19-953241-4, 2010)

[2]. M. Auzinsh, D. Budker, S.M. Rochester, Optically Polarized Atoms: Understanding LightAtom Interactions (Oxford University Press, ISBN 978-0-19-956512-2, 2010).

[3] S.V. Kargapoltsev, J. Kitching, L. Hollberg, A.V. Taichenachev, V.L. Velichansky, V.I. Yudin, Las. Phys. Lett. 1, 495 (2004).

[4] P. Tremblay, A. Nichaud, M. Levesque, S. Thériault, M. Breton, J. Beaubien, N. Cyr, Phys. Rev. A 42, 2766 (1990).

[5] E. B. Alexandrov, M.P. Chaika, G.I. Khvostenko, Interference of Atomic States (SpringerVerlag, 1993).

[6] A. Sargsyan, G. Hakhumyan, A. Papoyan, D. Sarkisyan, A. Atvars, M. Auzinsh, Appl. Phys. Lett. 93, 021119 (2008).

[7] A. Sargsyan, D. Sarkisyan, A. Papoyan, Y. Pashayan-Leroy, P. Moroshkin, A. Weis, A. Khanbekyan, E. Mariotti, L. Moi, Las. Phys. 18, 749 (2008).

[8] G. Dutier, A. Yarovitski, S. Saltiel, A. Papoyan, D. Sarkisyan, D. Bloch, M. Ducloy, Europhys. Lett. 63, 35 (2003).

[9] C. Andreeva, S. Cartaleva, L. Petrov, S.M. Saltiel, D. Sarkisyan, T. Varzhapetyan, D. Bloch, M. Ducloy, Phys. Rev. A 76, 013837 (2007).

[10] E.A. Gazazyan, A.V. Papoyan, D. Sarkisyan, A. Weis, Las. Phys. Lett. 4, 801 (2007).

[11] G. Hakhumyan, C. Leroy, R. Mirzoyan, Y. Pashayan-Leroy, D. Sarkisyan, Eur. Phys. J. D 66, 119 (2012).

[12] J. Keaveney, A. Sargsyan, U. Krohn, D. Sarkisyan, I.G. Hughes, C.S. Adams, Phys. Rev. Lett. 108, 173601 (2012).

[13] A. Sargsyan, G. Hakhumyan, C. Leroy, Y. Pashayan-Leroy, A. Papoyan, D. Sarkisyan, Opt. Lett. 37, 1379 (2012).

[14] B.A. Olsen, B. Patton, Y.-Y. Jau, W. Happer, Phys. Rev. A 84, 063410 (2011).

[15] L. Weller, K.S. Kleinbach, M.A. Zentile, S. Knappe, Ch.S Adams, I.G. Hughes, Opt. Lett. 37, 3405 (2012).

[16] A. Sargsyan, R. Mirzoyan, A. Papoyan, D. Sarkisyan, Opt. Lett. 37, 4871 (2012).

[17] L. Weller, K.S. Kleinbach, M.A. Zentile, S. Knappe, Ch.S. Adams, I.G. Hughes, J. Phys. B: At. Mol. Opt. Phys. 45, 215005 (2012).

[18] A. Sargsyan, R. Mirzoyan, D. Sarkisyan, JETP Lett. 96, 303 (2012).

[19] M. Fleischhauer, A. Imamoglu, J. P. Marangos, Rev. Mod. Phys. 77, 633 (2005). 